\section{A need for new imaging modality to detect inflammation in rheumatoid arthritis and osteoarthritis?}

\author{
Ida K Haugen, Hilde B Hammer
}

Optical spectral transmission (OST) and fluorescence optical imaging (FOI) represent new imaging modalities for assessment of inflammation in systemic inflammatory rheumatic diseases and osteoarthritis (OA). ${ }^{1-3}$

We know that inflammation is the primary pathogenic event in rheumatoid arthritis (RA) leading to joint damage and pain, if not treated. Hence, to optimise medical treatment in patients with RA, it is important to correctly assess the presence of inflammation. In OA, which was originally considered as a non-inflammatory disease, multiple studies have shown that inflammation is frequently present, both in early and later stages of the disease. ${ }^{4} 5$ Inflammation in osteoarthritic joints is often associated with pain and predicts future structural progression. ${ }^{5-10}$ Due to the limited number of clinical trials in OA, we have little knowledge whether reducing inflammation in OA leads to less structural progression in these patients. ${ }^{11}$

Sensitive and specific imaging modalities are important for assessment of inflammation, as clinical examination is hampered by low sensitivity and fair reliability. Both in research and clinical rheumatologic practice, inflammation in tendons and joints can be visualised using MRI and ultrasound. In RA, MRI and ultrasound are well established imaging modalities used in observational studies and clinical trials. The amount of research using MRI and ultrasound in hand $\mathrm{OA}$ is more limited, and further research is needed before the methods can be recommended as outcome measures in clinical trials. ${ }^{12}$

Do we need new imaging modalities for assessment of inflammation in rheumatology research and clinical practice in addition to those that are already available? MRI is hampered by the high cost related to the purchase, acquisition and reading, the limited availability in many centres and the contraindications related to acquisition

Department of Rheumatology, Diakonhjemmet Hospital, Oslo, Norway

Correspondence to Dr Ida K Haugen, Department of Rheumatology, Diakonhjemmet Hospital, Postboks 23 Vinderen, Oslo NO-0319, Norway; ida.k.haugen@gmail.com and the contrast agent. Using extremity or portable MRI scanners the costs can be lowered and certain contraindications can be circumvented. The lower field strength cannot support as much image resolution or as many contrast mechanisms as conventional whole-body 1.5 or 3 Tesla systems. However, they may be useful to detect joint inflammation. ${ }^{13}$ Ultrasound on the other hand is more easily available in rheumatology clinics with low costs related to the actual scanning. The main disadvantage of ultrasound is the operator dependency related to the interpretation of the images and the actual performance of the scanning. However, several studies have shown high reliability for scanning, and the more common use of high-end ultrasound machines may improve reliability in the clinical setting. Whereas longitudinal MRIs can be evaluated pairwise, evaluation of change in observational studies as well as clinical trials may be more challenging using ultrasound.

The introduction of new imaging methods to assess joint inflammation in RA and OA are thus welcomed. However, it is of major importance to evaluate the feasibility, reliability and validity of all new modalities to ascertain good quality. Two devices have been launched: HandScan (based on OST) and Xiralite (also called the Rheumascan, based on FOI).

OST and FOI are feasible methods, which can be performed by trained personnel (eg, nurses). The advantage of OST and FOI is the short acquisition time of $90 \mathrm{~s}$ and $6 \mathrm{~min}$, respectively. Furthermore, the OST has the benefit of being a non-invasive method, based on the transmission of light before and after impeding the venous return of blood from the fore arms. The disadvantage of FOI is the required administration of an intravenous fluorescence dye (Indocyanine Green, ICG-Pulsion), which should not be given to patients with poor liver function, allergies to shellfish or hyperthyroidism due to the content of iodine. Hence, blood screening is mandatory prior to examination. Another benefit of the OST is the automatic generation of a quantitative inflammatory score for each joint using a pre-defined algorithm based on the absorption of light. Similar to ultrasound and MRI, the evaluation of FOI images requires a semi-quantitative subjective evaluation of the fluorescence signal intensity on 0-3 scales. The evaluation requires training, but should be possible to learn by not only rheumatologists, but also nurses. Overall, OST and FOI seem to be feasible methods, but one should bear in mind that the FOI requires the use of intravenous fluorescence dye and training of readers. One should be aware that the costs related to purchase are relatively large (somewhere between the cost of an ultrasound scanner and an MRI scanner). In addition, the performance of FOI requires the use of fluorescence dye, which is an additional cost.

When it comes to reliability, no information is provided in any of the three published studies. ${ }^{1-3}$ Especially for semiquantitative subjective evaluation of FOI, reliability is of major importance. One would expect that the automatic generation of OST scores would lead to high reliability, but actual numbers to prove this assumption were not presented.

The three studies presented in the current volume focus on the validity of optical imaging in RA and OA. Whereas FOI has been tested in a number of studies on inflammatory arthritic diseases, ${ }^{14-17}$ we have limited knowledge about the performance of the OST. Van Onna et al ${ }^{1}$ explored the performance of OST using ultrasound and MRI as reference in patients with RA and arthralgia (controls). Patients with RA were divided into groups with increasing levels of disease activity scores (DAS28), and increasing disease activity scores were associated with higher OST values. OST was found to have only moderate agreement with clinical examination, ultrasound and MRI assessments, and performed better in the proximal interphalangeal (PIP) and metacarpophalangeal (MCP) joints than the wrists. Most importantly, their results suggested that OST was a more sensitive method to detect joint areas with inflammation than clinical examination. ${ }^{1}$ However, Krabbe et al ${ }^{2}$ did not confirm the high sensitivity of OST to detect inflammation, questioning the usefulness of the method and the validity of the results. The sensitivity and specificity to detect inflammation by OST will vary depending on the pre-defined cut-off value for presence of inflammation. Van Onna et $a l^{1}$ used the same study sample for development and validation of the algorithm to be used for calculation of the inflammatory score, which may have overestimated the sensitivity of the method. Using the same cut-off values in a separate cohort, Krabbe 
et $a l^{2}$ did not show any benefit of OST in comparison to clinical examination. These results suggest that further development of the algorithm is needed before OST can become a useful tool for quick, operatorindependent, non-invasive evaluation of joint inflammation in RA.

The paper by Glimm et $a l^{3}$ is the first study exploring the use of optical imaging in patients with hand OA. The amount of inflammation using FOI in hand OA was comparable to RA, although differences were found in the different phases of fluorescence uptake. Looking at the composite image, which is based on the 240 first images (of 360 in total), patients with OA demonstrated more inflammation in the distal interphalangeal and PIP joints compared with patients with RA, which is expected based on the joint involvement in OA. More surprisingly, the number of patients with inflammation in the MCP and wrist joints was almost identical across RA and OA, and the wrist was the most commonly affected joint in both diseases. ${ }^{3}$ Based on the very low prevalence of radiographic OA in the wrist joint shown in the Framingham study, ${ }^{18}$ these findings are surprising and may question the validity of the FOI findings. Exploring the different phases of fluorescence signal intensities, more inflammation was found in patients with RA in the earlier phase, suggesting more active inflammation in the wrist, MCP and PIP joints in RA. In contrast, patients with $\mathrm{OA}$ demonstrated stronger fluorescence signal intensities in the wrists and PIP joints in the later phases, possibly related to joint damage. ${ }^{3}$ Hence, FOI may be able to detect different inflammatory patterns in rheumatic diseases. The main conclusion and important message of the study is the strong involvement of inflammation in OA. ${ }^{3}$ However, the limitations of this study are the lack of sensitivity and specificity values of FOI in comparison to ultrasound, even though ultrasound examination was part of the study. Furthermore, patients with RA had moderate disease activity, but no information about their treatment was provided, for example, the use of corticosteroids. According to earlier studies and clinical experience, power Doppler activity, as a measure of vascularity, will decrease in patients on corticosteroids. ${ }^{19}$ It is therefore likely that FOI will also be affected by treatment.

To our knowledge, no previous studies have explored the use of optical imaging for monitoring treatment of arthritis, and future studies are needed to explore their sensitivity to change compared with other imaging modalities and clinical examination.

To optimise medical treatment in rheumatic diseases, it is important to correctly assess the presence of inflammation, and optical imaging modalities may be useful supplements. Up till now, the reliability and validity of these methods have not been sufficiently tested, and more research is therefore needed. As opposed to ultrasound and MRI, which provide a precise localisation of the inflammation, the differentiation between synovitis and tenosynovitis is more challenging using optical imaging. Furthermore, optical imaging provides information about inflammation only, and can never replace MRI or ultrasound due to the lack of evaluation of structural damage. However, new methods must also be feasible, and this is fulfilled by only a few minutes to perform the FOI and OST examinations.

Contributors IKH: drafting the work; final approval of the version published. $\mathrm{HBH}$ : revising the work critically for important intellectual content; final approval of the version published.

\section{Competing interests None declared.}

Provenance and peer review Commissioned; externally peer reviewed.

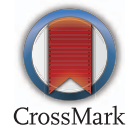

To cite Haugen IK, Hammer HB. Ann Rheum Dis 2016;75:479-480

Received 25 September 2015

Revised 28 November 2015

Accepted 30 November 2015

Published Online First 23 December 2015

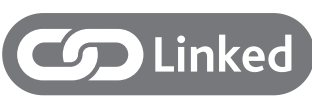

- http://dx.doi.org/10.1136/annrheumdis-2015207345

- http://dx.doi.org/10.1136/annrheumdis-2015207315

http://dx.doi.org/10.1136/annrheumdis-2015208399

Ann Rheum Dis 2016:75:479-480. doi:10.1136/annrheumdis-2015-208661

\section{REFERENCES}

1 van Onna M, Ten Cate D, Tsoi KL, et al. Assessment of disease activity in patients with rheumatoid arthritis using optical spectral transmission measurements, a non-invasive imaging technique. Ann Rheum Dis 2016;75:511-18.

2 Krabbe S, Ammitzbøll-Danielsen M, Østergaard M, et al. Sensitivity and specificity of optical spectral transmission imaging in detecting joint inflammation in rheumatoid arthritis. Ann Rheum Dis 2016;75:632-3.

3 Glimm AM, Werner SG, Burmester GR, et al. Analysis of distribution and severity of inflammation in patients with osteoarthritis compared to rheumatoid arthritis by ICG-enhanced fluorescence optical imaging and musculoskeletal ultrasound: a pilot study. Ann Rheum Dis 2016:75:566-70.

4 Haugen IK, Bøyesen P, Slatkowsky-Christensen B, et al. Comparison of features by MRI and radiographs of the interphalangeal finger joints in patients with hand osteoarthritis. Ann Rheum Dis 2012:71:345-50.

5 Atukorala I, Kwoh CK, Guermazi A, et al. Synovitis in knee osteoarthritis: a precursor of disease? Ann Rheum Dis 2016:75:390-5.

6 Haugen IK, Bøyesen P, Slatkowsky-Christensen B, et al. Associations between MRI-defined synovitis, bone marrow lesions and structural features and measures of pain and physical function in hand osteoarthritis. Ann Rheum Dis 2012;71:899-904.

7 Haugen IK, Slatkowsky-Christensen B, Bøyesen P, et al. MRI findings predict radiographic progression and development of erosions in hand osteoarthritis. Ann Rheum Dis 2016;75:117-23.

8 Haugen IK, Slatkowsky Christensen B, Bøyesen P, et al. Increasing synovitis and bone marrow lesions are associated with incident joint tenderness in hand osteoarthritis. Ann Rheum Dis 2015. Published Online First 9 Mar 2015. doi:10.1136/annrheumdis2014-206829

9 Mathiessen A, Slatkowsky-Christensen B, Kvien TK, et al. Ultrasound-detected inflammation predicts radiographic progression in hand osteoarthritis after 5 years. Ann Rheum Dis 2015. Published Online First 1 Apr 2015. doi:10.1136/annrheumdis-2015-207241

10 Yusuf E, Kortekaas MC, Watt I, et al. Do knee abnormalities visualised on MRI explain knee pain in knee osteoarthritis? A systematic review. Ann Rheum Dis 2011:70:60-7.

11 Verbruggen G, Wittoek R, Vander Cruyssen B, et al. Tumour necrosis factor blockade for the treatment of erosive osteoarthritis of the interphalangeal finger joints: a double blind, randomised trial on structure modification. Ann Rheum Dis 2012;71:891-8.

12 Kloppenburg M, Bøyesen P, Visser AW, et al. Report from the OMERACT hand osteoarthritis working group: set of core domains and preliminary set of instruments for use in clinical trials and observational studies. J Rheumatol 2015;42:2190-7.

13 Peterfy CG. Is there a role for extremity magnetic resonance imaging in routine clinical management of rheumatoid arthritis? J Rheumatol 2004:31:640-4.

14 Krohn M, Ohrndorf S, Werner SG, et al. Near-infrared fluorescence optical imaging in early rheumatoid arthritis: a comparison to magnetic resonance imaging and ultrasonography. J Rheumatol 2015:42:1112-18.

15 Werner SG, Langer H-E, Schott P, et al. Indocyanine green-enhanced fluorescence optical imaging in patients with early and very early arthritis: a comparative study with magnetic resonance imaging. Arthritis Rheum 2013;65:3036-44.

16 Schäfer VS, Hartung W, Hoffstetter P, et al. Quantitative assessment of synovitis in patients with rheumatoid arthritis using fluorescence optical imaging. Arthritis Res Ther 2013;15:R124.

17 Werner SG, Langer H-E, Ohrndorf S, et al. Inflammation assessment in patients with arthritis using a novel in vivo fluorescence optical imaging technology. Ann Rheum Dis 2012;71:504-10

18 Haugen IK, Englund M, Aliabadi P, et al. Prevalence, incidence and progression of hand osteoarthritis in the general population: the Framingham Osteoarthritis Study. Ann Rheum Dis 2011;70:1581-6.

19 Larché MJ, Seymour M, Lim A, et al. Quantitative power Doppler ultrasonography is a sensitive measure of metacarpophalangeal joint synovial vascularity in rheumatoid arthritis and declines significantly following a 2-week course of oral low-dose corticosteroids. J Rheumatol 2010;37:2493-501. 